\title{
Division of the External Urethral Sphincter in the Treatment of the Paraplegic Bladder: A Preliminary Report on a New Procedure
}

\author{
J. Cosbie Ross, Ch.M., F.R.C.S., N. O. K. Gibbon, Ch.M., F.R.C.S. and \\ M. Damanski, M.D. \\ Liverpool Regional Paraplegic Centre, Southport, England
}

It is a little-known fact that certain patients suffering from neurogenic bladder may be finally left with an apparently unsurmountable obstruction at the level of the external urethral sphincter, despite abolition of reflex activity in that region by intrathecal alcohol block or pudendal neurectomy (Cosbie Ross et al., $1957 a, b$ ). In certain patients with quadriplegia abdominal straining is weak or absent owing to loss of power of the abdominal muscles. In others the detrusor shows no evidence of reflex activity as a result of previous subarachnoid alcohol blocks given for spasticity of the legs.

The situation may not arise often, but in any given group of patients with paraplegia there will remain a small percentage, a hard core, in which alcohol block, resection of the bladder neck, and pudendal neurectomy have failed to produce an efficient automatic bladder. To illustrate that this is not a negligible problem, reference must be made to the recent paper by Bors (1956) in which $80^{\circ} \%$ of patients with quadriplegia, still in hospital, remained on permanent catheter drainage. It is clear that something new had to be found to prevent such an undesirable sequel. The conception that the obstruction may be at the external sphincter level is not new. Watkins (1936) found that in some cauda equina lesions the critical obstruction lay at the level of the urogenital diaphragm and he blamed deformity and lack of elasticity in that region for difficult bladder emptying. Whether his original view was correct or not, it remains a fact that the localisation of organic obstruction at the external sphincter level seems to apply to-day in some cases of paraplegia. Cystographic studies carried out on a large number of paraplegic patients (Damanski and Kerr, 1957) have confirmed this theory. It is only in recent years, as a result of the failure of pudendal neurectomy to relieve an external sphincter obstruction in certain cases (Cosbie Ross, 1956), that Watkins' observation has come again into prominence.

The operation of division or resection of the external sphincter was first described by one of us (J. C. R.) in 1956, and we believe that the procedure has not previously been used for this particular purpose.

Reprinted from the British Journal of Urology, Vol. XXX, No. 2, June 1958, by kind permission of the British Association of Urological Surgeons. 


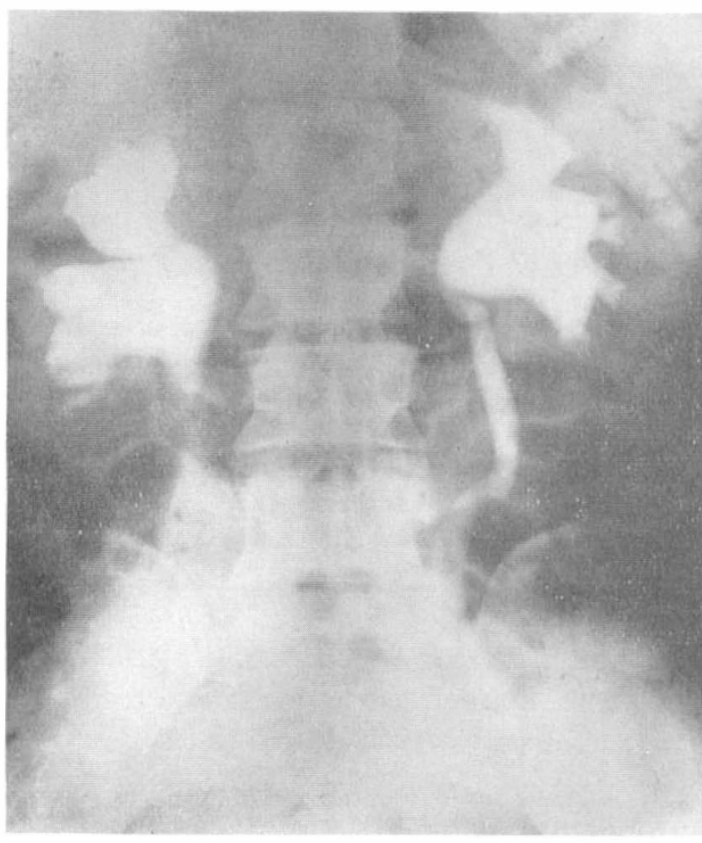

$\lambda$

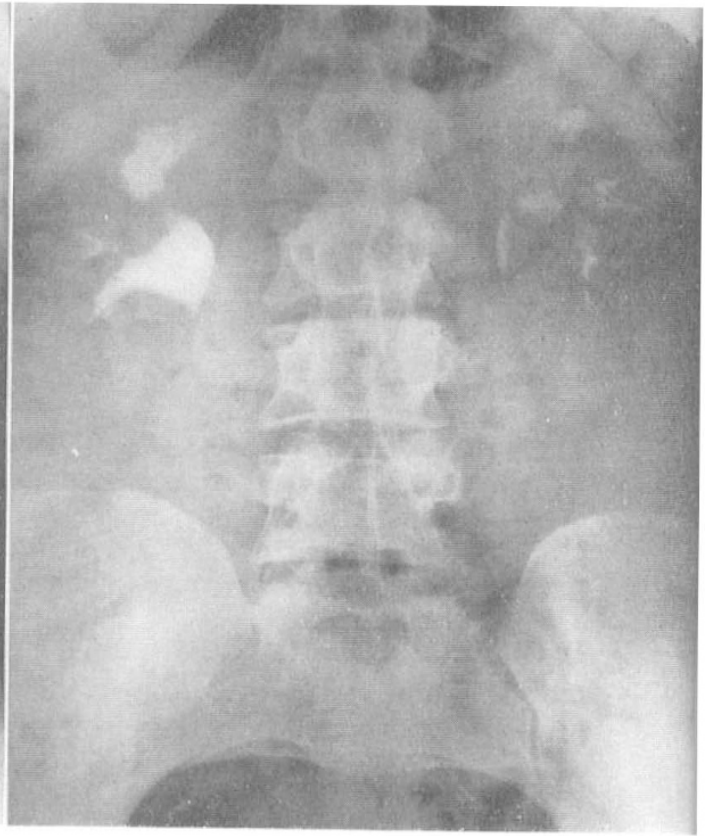

B

Figure 1 Intravenous pyelograms.

A. Pre-operative. Note the high degree of bilateral hydronephrosis and hydro-ureter.

B. Post-operative. The left upper tract is now normal. The right side is a little dilated.

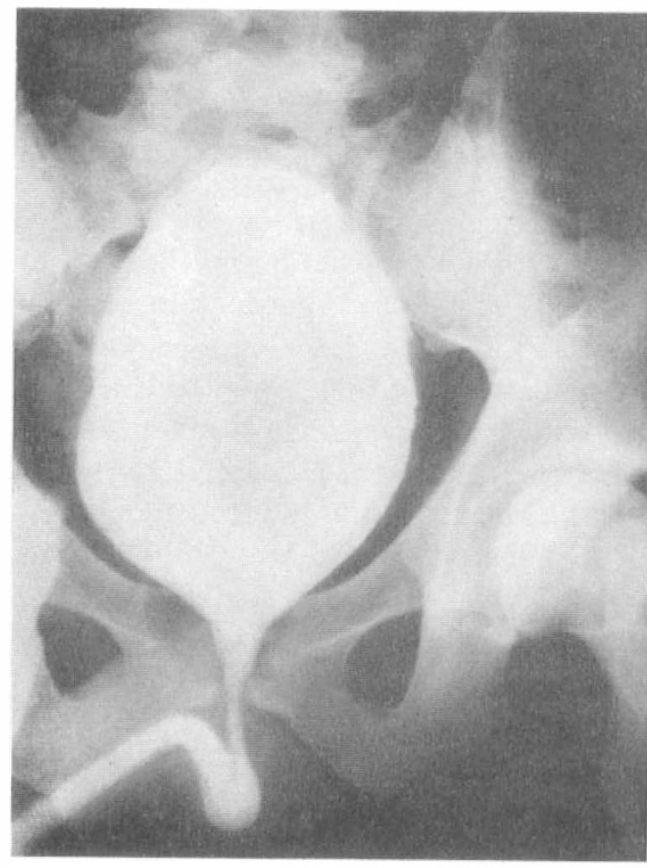

A

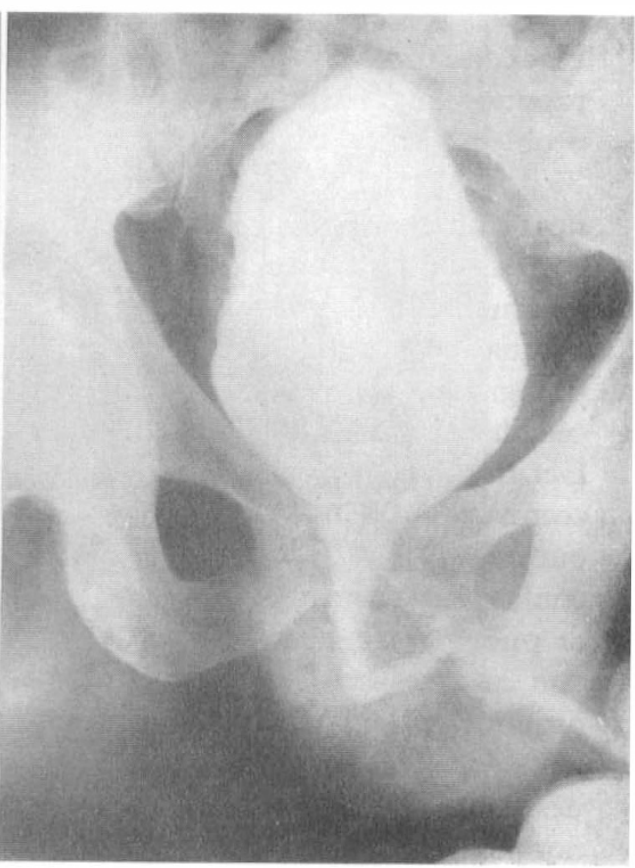

B

Figure 2 Cysto-urethrograms. Retrograde filling, anteroposterior view.

A. Pre-operative. The bladder neck, the prostatic and membranous urethra show moderate degree of relaxation when filled from below.

B. Post-operative. Note how more widely the same structures open in similar conditions. 
Before going into further details of the clinical picture and the suggested treatment, we are presenting short summaries of ten patients illustrating the problem.

Case 1.-J. H., aged 22. Fracture-dislocation C6 on C7. Spastic quadriplegia at the level C8. Two subarachnoid alcohol blocks were given for spasticity. A high residual urine and an autonomic reaction to bladder distension (such as headache and feeling of throbbing, patchy redness of skin mainly above the level of paralysis and congestion of face, prof use perspiration and cutis anserina) remained unaffected by the blocks. A bladder-neck resection lowered the residual urine for a short time only; it accrued later, although not to the previous level. Another two blocks were performed later for a return of spasticity. The patient developed bilateral hydronephrosis in the meantime (Fig. 1, A and B), the high residual urine remaining unchanged.

Case 2.-L. H., aged 36. Fracture-dislocation C6 on C7. Spastic quadriplegia at the level of C6 to $\mathrm{C} 7$. An alcohol block had abolished spasticity permanently and also diminished the autonomic hyperreflexia. Complete retention of urine persisted together with reflux up the left ureter (Fig. 2, $A$ and $B)$.

Case 3.-T. M., aged 20. Fracture-dislocation C5 on C6. Quadriplegia at the level of C7. Considerable spasticity and autonomic hyperreflexia. Had an alcohol block which helped his spasticity and autonomic hyperreflexia but did not relieve the retention of urine. There was also reflux to the right kidney.

Case 4.-H. S., aged 60. Fracture-dislocation C4 on C5. Quadriplegia at the level of C7. Considerable spasticity necessitated three alcohol blocks after which the left leg still retained a moderate degree of spasticity. A high residual urine remained unaffected. When in a general hospital developed a large stone in the right ureter, with hydronephrosis, and a larger one in the bladder.

Case 5.-J. D., aged 20. Thrombosis of the anterior spinal artery causing considerable spastic quadriplegia at the level of $\mathrm{C} 7$. Had two alcohol blocks as a result of which spasticity was diminished but not abolished and a high residual urine persisted.

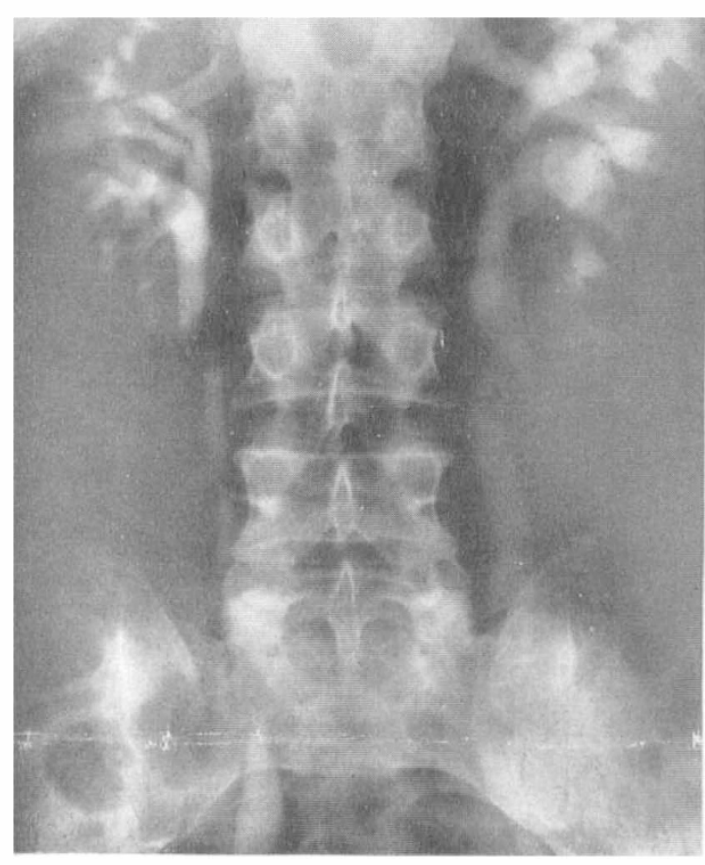

1

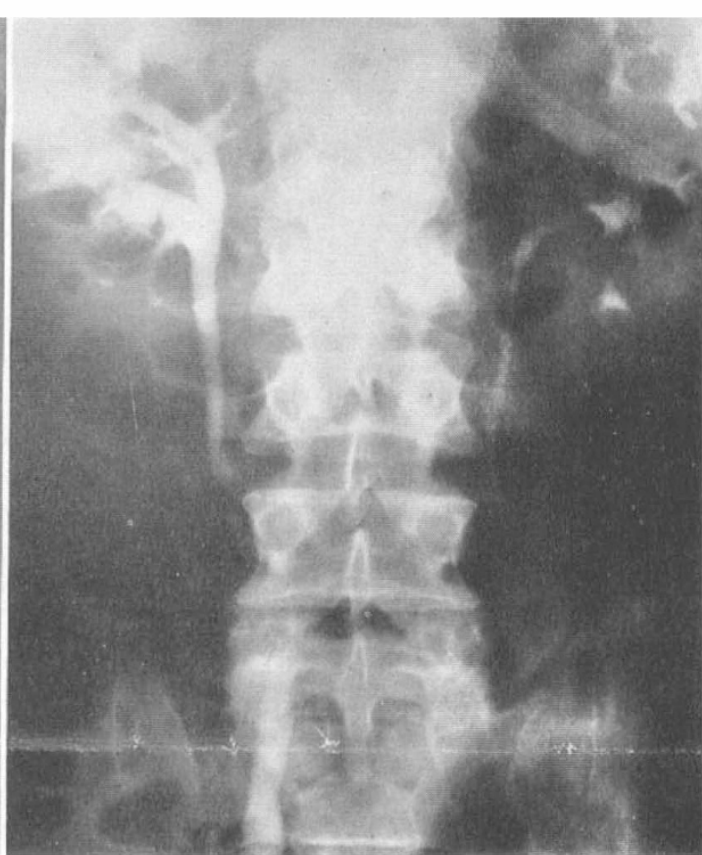

B

Figure 3 Intravenous pyelograms.

A. Pre-operative. Note bilateral dilatation of the upper tract, more so on the left.

B. Post-operative. The left tract is now normal, the right one is still dilated. 


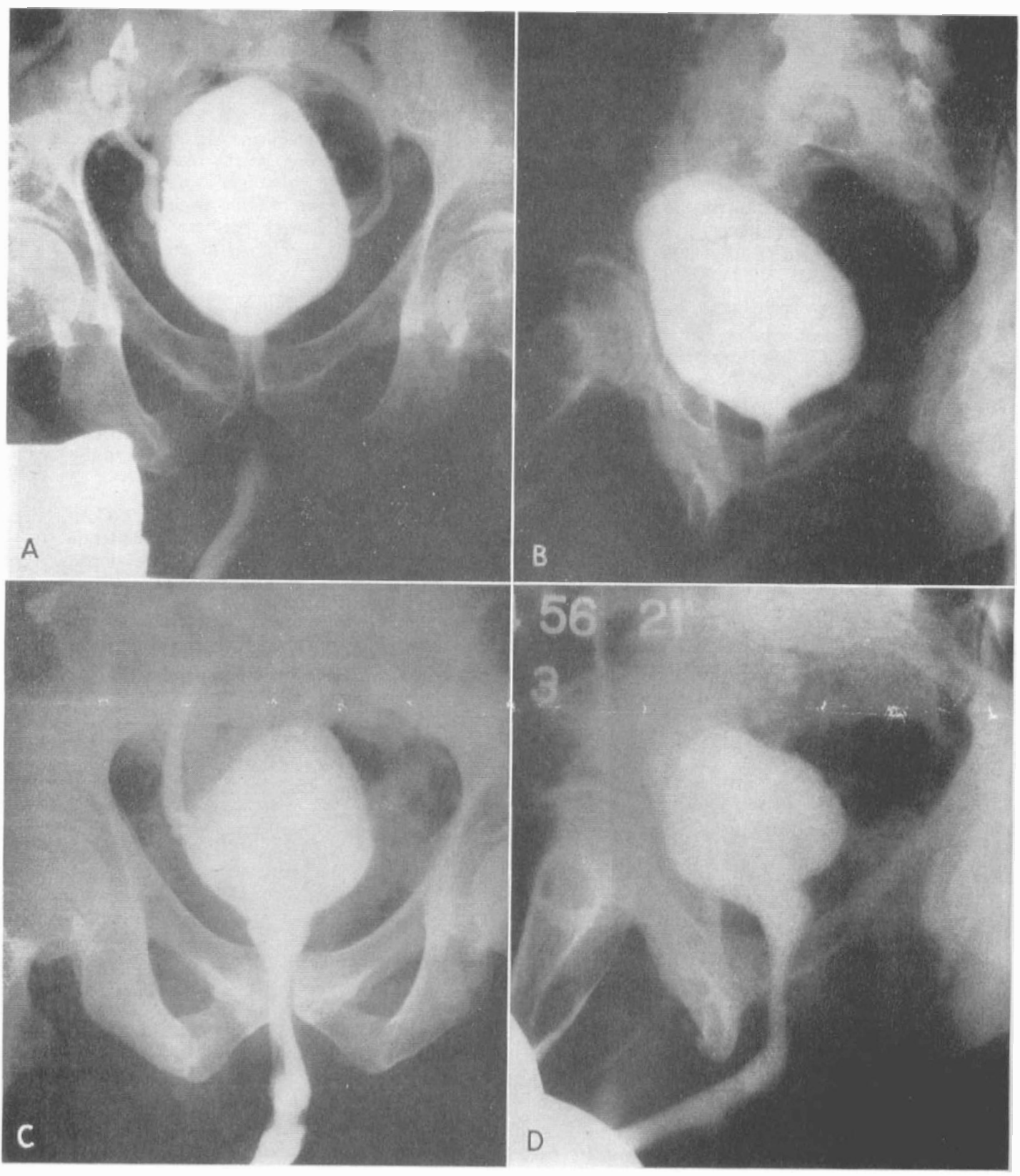

Figure 4 Cysto-urethrogram.

A. Cysto-urethrogram, retrograde filling, anteroposterior view, pre-operative film. Note incomplete filling of the proximal prostatic urethra and the backflow to both lower ureters.

B. Cysto-urethrogram, retrograde filling, oblique view, pre-operative film. Similar appearance. Note also the median bar.

C. Anteroposterior view. Note how widely the whole posterior urethra has opened. There is still backflow into the right lower ureter.

D. Oblique view. Similarly wide opening of the posterior urethra. 
Case 6.-J. P., aged 39. Disseminated sclerosis in an advanced stage with considerable spasticity typical of the neurological condition. There was complete retention of urine with secondary dilatation of the upper tract. An alcohol block was out of the question owing to incompleteness of the neurological lesion. Pudendal neurectomy and bladder-neck resection both resulted in failure.

Case 7.-J. S., aged 32. Fracture-dislocation T11 on T12. Paraplegia at the level of T11. Slight spasticity. Residual urine was reduced temporarily by a pudendal neurectomy. Patient was discharged home prematurely and developed considerable hydronephrosis and hydro-ureter (Fig. 3, A and $\mathbf{B})$.

Case 8.-T. N., aged 49. Fracture-dislocation T11 on T12. Paraplegia at the level of L1. Slightly spastic. A high residual urine despite a bladder-neck resection and a pudendal neurectomy. There was reflux up both ureters (Fig. 4, A, B, C and D).

Case 9.- J. B., aged 36. G. S. W., L3. Paraplegia at the level of L1 to L2. Bilateral hydronephrosis and massive backflow to the distended upper tracts. The residual urine was $19 \mathrm{oz}$. in spite of pudendal neurectomy.

Case 10.-N. P. R. C., aged 46. Spastic paraplegia T12 owing to meningomyelitis twelve years previously. He was originally treated in other hospitals, being admitted to our centre for gangrene of a leg. He had previously had a bladder-neck resection performed in the early stage of paraplegia. There was a high residual urine with cavitations of the prostate resulting from infection. There was no improvement after pudendal neurectomy (Fig. 5, A and B).

\section{All these patients had the following features in common:}

1. Retention of urine, complete or incomplete, despite the previous procedures.

2. In spite of the elimination of spasticity by previous procedures it was possible to demonstrate a definite obstruction at the level of the external

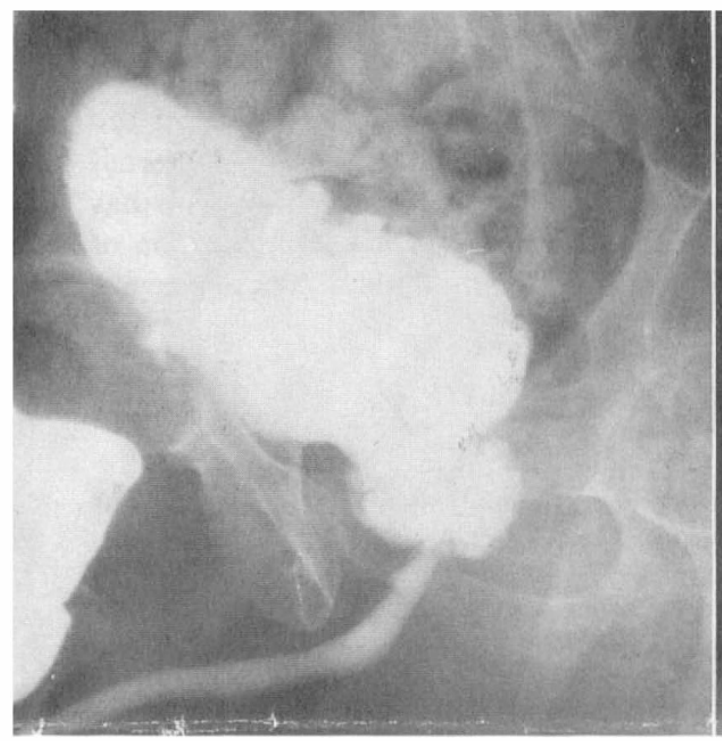

A

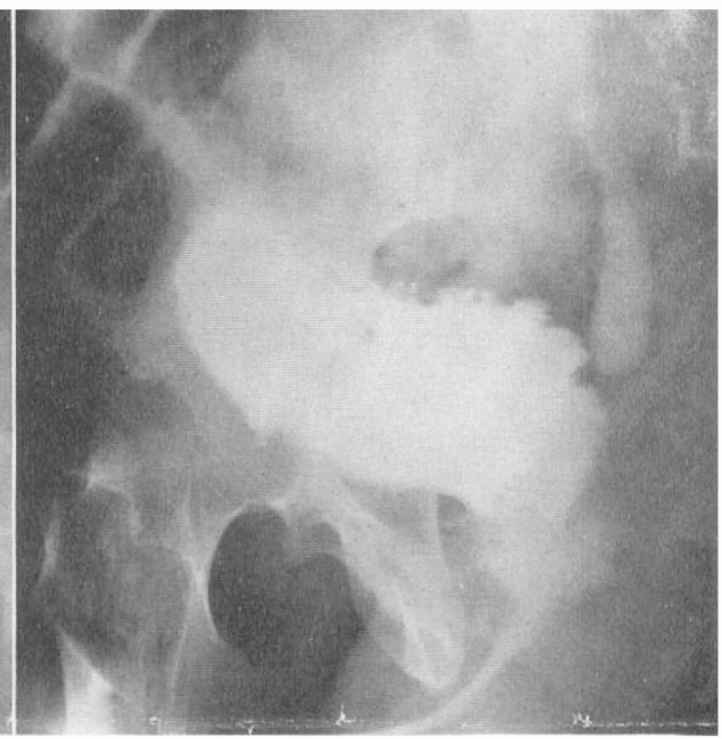

B

Figure 5

A. Cysto-urethrogram, retrograde filling, oblique view, pre-operative film. Note the narrow passage at the external sphincter. The bladder neck is open and the prostatic parenchyma is considerably destroyed by the infection of long standing.

B. Cysto-urethrogram, retrograde filling, oblique view, post-operative film. Note wide opening of the previously strictured area. The prostatic cavities appear to be less filled with contrast medium. 
sphincter, certainly by cysto-urethrography and sometimes by cystourethroscopy.

3. Heavy infection of urine of several months' or years' (Case 10) standing.

All ten patients have been given the benefit of transurethral resection of tissue from the external sphincter area, the surviving eight cases with a most satisfactory result.

The idea of attacking the recalcitrant external sphincter surgically is not new. Donovan (1949) suggested that extensive punching-out of the obstruction at the level of the urogenital diaphragm should be carried out, but he feared that this would be followed by incontinence of urine.

It is the purpose of this paper to report on our method of division or resection (whichever term be more acceptable) of the external urethral sphincter in the treatment of the patients described above.

\section{Indications}

If a quadriplegic or paraplegic patient has complete or incomplete retention due to obstruction at the external sphincter level, then an attempt should be made to relieve him by subarachnoid alcohol block (Shelden and Bors, 1948) or by pudendal neurectomy (Cosbie Ross and Damanski, 1953; Bors and Comarr, 1954; Cosbie Ross, 1956) provided his conus-reflex activity is maintained. Should these procedures fail we contend that resection of the external sphincter should be considered. Ureteric backflow, diverticulosis, or trabeculation of the bladder, filling of the prostatic ducts at cysto-urethrography (Damanski and Gibbon, 1956), and frequent exacerbations of the chronic urinary infection make the procedure more urgent.

Further experience may modify this somewhat cautious approach and the indications may be extended. We must emphasise that division of the external sphincter should be reserved for the patient with chronic retention who has failed to respond to more orthodox methods such as alcohol block, resection of the bladder neck, and pudendal neurectomy. It is a method to be used to prevent a catheter life in these difficult patients.

\section{Contraindications}

Our experience at the moment is too limited to establish these categorically, but we consider:

1. A heavy urinary infection which cannot be controlled by antibiotics or continuous bladder drainage, at least in the immediate pre-operative and postoperative period.

2. An advanced stage of hydronephrosis.

In certain quadriplegic patients who cannot exert manual or abdominal pressure upon the bladder, and in whom reflex vesical activity is absent, efficient bladder-emptying cannot be expected, however low the resistance to voiding. Permanent drainage by nipple ureterostomy or transplant of the ureters into a segregated ileal loop may be necessary. 


\section{Pre-operative care}

An appropriate antibiotic should be administered twenty-four to forty-eight hours prior to the operation and an indwelling Gibbon's (1958) polyvinyl chloride tube inserted to keep the infection in check. Daily bladder washouts may be of assistance. Blood should be readily available for a transfusion or transfusions.

\section{Technique}

We have used the cold punch in almost all of our cases, resecting several strips from the external sphincter, usually posterolaterally. In one patient, after failure of this procedure, the external sphincter was exposed and divided by an open perineal approach, but again without improvement. Repetition of the perurethral operation, however, was eventually successful. In some instances the procedure was associated with a resection of a few strips from the bladder neck but it was considered that this additional resection did not essentially affect the issue in view of the widely open bladder neck. On one occasion, when the operation had to be repeated, the external sphincter was divided using a Colling's knife through a urethroscope. It may well be that this will prove the safest and most efficient method.

The resection may open up small venous sinuses, and we have not found diathermic haemostasis to be effective. We therefore insert a large catheter (Charrière 24 to 28 ) immediately on completion of the resection in order to control the haemorrhage by pressure.

Addendum. In order to ensure greater safety during the post-operative period we have recently divided the external sphincter by diathermy through an external urethostomy approach in three patients. It is too early, as yet, to attempt an assessment of this method.

\section{Post-operative care}

It cannot be sufficiently stressed that the majority of paraplegic patients have a more severe reaction to any procedure on the bladder neck than those with a normal innervation. Two reasons are responsible for this reaction:

1. Neurological changes affecting the contractibility of the blood-vessels (bleeding time). To our knowledge no such studies have been carried out, but this is a generally recognised fact.

2. The presence of a chronic urinary infection.

The same applies in a still higher degree to the external sphincter area. Postoperative care is most important and continuous observation is necessary for several hours in order to prevent, or to deal quickly with, clot retention. The type of catheter used is not of great importance and either a 'three-way' or one with several lateral holes and a terminal orifice may be inserted. It also makes no substantial difference whether continuous or syringe irrigation is carried out. What matters most is a sound clinical judgment which must be exercised in assessing whether there is a satisfactory return and whether the bladder is dis- 
tended. This is not an easy decision. Palpation of the distended bladder may be difficult owing to spasticity of the abdominal musculature in cases of spastic paraplegia. An increase or reappearance of autonomic hyperreflexia (as described in Case 1), vomiting, and symptoms of haemorrhage and shock are the most helpful signs of clot retention.

The patient's position in bed must be changed two-hourly to four-hourly. The more he bleeds the more often this should be done, as haematuria brings about a drop in the plasma-protein level and renders the patient more liable to pressure sores. Depletion of plasma proteins must be corrected by blood transfusion.

The patient's bed must be kept scrupulously dry.

The large-size catheter is changed to a Gibbon's polyvinyl chloride tube or a latex catheter (No. 14 to $15 \mathrm{~F}$ ) as soon as the gross haematuria has cleared. The change should be carried out at the first possible moment.

Digital evacuation of the rectum should be done daily and as gently as possible. Too vigorous a manipulation disturbs the adjoining post-operative wound.

Antibiotic treatment is continued as long as sensitivity tests show that it is still effective.

\section{Complications}

The post-operative haematuria may be more severe than that after a bladderneck resection. One patient had to have a suprapubic cystostomy established temporarily twenty-four hours after the operation. In three other patients a secondary haemorrhage occurred and two of them had to be taken to the operating theatre to have the clots evacuated. This may have been due to our inexperience when dealing with the first group of patients. In one fatal case, haematuria recurred repeatedly over a period of a fortnight and death occurred on the operating table during the performance of a suprapubic cystostomy. We think that this patient's life might have been saved if suprapubic drainage had been carried out earlier.

We also saw swelling of the scrotum due to extravasation of blood, and possibly of the fluid used for irrigation, during the operation in one case, but it disappeared shortly after a pressure-dressing had been applied. In another case it appeared simultaneously with a clot retention twenty-four hours after the operation. Perineal incisions were made, but we wonder whether this radical approach was necessary.

\section{Mechanism of action and pathology}

In none of the operated cases was there any reason to assume that the previously attempted denervation of the muscles of the bladder neck and the external sphincter had been incomplete. The bulbocavernosus reflex had been abolished by previous alcohol block or by pudendal neurectomy. Biopsy of the resected strips have shown either infiltration by chronic inflammatory cells, or inflammation and fibrosis (Cases 5, 8 and 10).

It is felt that the muscular fibres had lost their contractibility, resulting in 
rigidity and a form of stricture. In support of this contention it may be mentioned that when a cystoscope or a metal bougie is passed on a paraplegic patient there is frequently a stiffness and lack of elasticity extending from the urogenital diaphragm to the bladder neck. The operation is, in effect, an internal urethrotomy.

\section{Assessment of results}

\section{Function}

Out of ten patients the result was eminently satisfactory in eight. Of the eight successful cases five had their residual urines reduced to negligible proportions (Cases 1, 4, 6, 7 and 10) and in the remaining three it has been reduced to 6 to 8 fl. oz. It seems likely that the effect of the operation on these three patients might be improved by a repetition of the procedure. A typical example is presented by Case 3 who was a failure after the first transurethral procedure, did not react at all to the perineal procedure (see under Technique), but has responded well to the second transurethral resection. He is a quadriplegic, and it must be remembered that these patients display weak abdominal straining so that it is essential that the resistance at the bladder neck and the external sphincter be reduced to a minimum. We would like to point out that, owing to the somewhat sharp post-operative reaction, considerable caution has been exercised and the amount of tissue resected was limited to approximately four strips in the earlier cases. It is evidently necessary to resect more, but this might be avoided by dividing the sphincter through a urethroscope with a Colling's knife. This procedure has been carried out recently with success. All the successful cases show stress incontinence which, far from being harmful, is beneficial. These patients all use a urine-collecting appliance in any event, and a penile clamp occasionally. The great benefit conferred by the operation is that eight patients have thereby been spared a catheter life with all its attendant dangers and difficulties.

\section{Infection}

We have obtained a sterile urine in one patient only (Case 4). In two the urinary infection has been considerably reduced. In the remaining five it is difficult to form an opinion as the appearance of the urine depends so much on the varying fluid intake and extrarenal factors. We hope that a more prolonged follow-up will throw some light on this aspect.

\section{Cysto-urethrographic findings}

Considerable widening of the urethral lumen in the external sphincter area has been obtained in four patients (Figs. 2, 4, and 5) and moderate widening in one patient (Case 2). Equivocal results were seen in two patients (Cases 3 and 6) and unchanged appearance in one (Case 7). The latter patient had a superficial resection with an excellent functional result and, in addition, the dilated upper urinary tract returned to normal. 
Ureteric backflow was originally present in five patients, disappearing after operation in two and remaining unchanged in two. The fifth had bilateral massive backflow and proved to be one of the two post-operative deaths in the series.

\section{Intravenous pyelography}

In one of the patients (Case 1), who had developed a marked bilateral hydronephrosis while waiting for operation, the changes cleared up completely on the left side and diminished considerably on the right during the post-operative period (Fig. 1).

In Case 7 a bilateral hydronephrosis and hydro-ureter were noted at the first follow up, but four months after the resection the upper urinary tract had returned to normal (Fig. 3).

One patient (Case 4) was admitted from a general hospital with a stone in the dilated upper ureter and a large stone in the bladder. Both were removed before the resection, the patient's upper tract returning to normal and his urine becoming sterile. The improvement here might be attributed to the effect of the removal of the stones. The remaining patients had normal intravenous pyelograms.

This is not a long-term survey similar to our previous report (Damanski and Gibbon, 1956), nevertheless the results are satisfactory up to the time of writing.

\section{Mortality}

One patient (Case 9) died on the third post-operative day from a violent exacerbation of urinary infection. Paper-thin renal parenchyma was found at the post-mortem examination and, no doubt, he would have died from uraemia in due course in any event. A sudden strain on the few remaining nephrons undoubtedly precipitated renal failure. In retrospect it is clear that division of the external sphincter should never have been attempted in this patient. The correct treatment here would have necessitated a tranplant of the ureters into a segregated ileal loop.

The other patient had a radiologically normal upper tract but a heavy urinary infection. During the post-operative period the haematuria was subsiding normally and his urine was almost clear from blood after three days. He then developed a stormy pyrexia with the return of haematuria. Chemotherapy and blood transfusions were unavailing. Clot retention recurred on several occasions and it was finally decided to establish suprapubic drainage, but the patient died during the operation. It is possible that this fatality could have been prevented had the suprapubic drainage been carried out earlier.

\section{Summary}

1. Division or resection of the external sphincter is a new operation designed to relieve retention of urine (or a high residue) in patients with an open bladderneck and a narrow, rigid external sphincter area and in whom subarachnoid alcohol block, pudendal neurectomy or a bladder-neck resection have failed to help. The operation converts a chronic retention to an easily expressible bladder. 
2. Ten patients are reported.

3. The operation technique is described.

4. During the post-operative period care is necessary in preventing or dealing with clot-retention and with severe infection due to absorption of organisms in the urethra.

5. The operation should not be attempted in patients with grossly dilated upper urinary tracts and in those with uncontrollable infection.

6 . The eight surviving patients have been spared the catheter life and, possibly, subsequent damage to the upper urinary tract.

\section{References}

Bors E 1956 Bulletin of the Los Angeles Neurological Society 21:105

BORS E, COMARR AE 1954 fournal of Urology 72:666

DAMANSKI M, GiBBON N 1956 British fournal of Urology 28:24

DAMANSKI M, KERR AS 1957 British fournal of Surgery 44:398

Donovan H 1949 Post-graduate Medical fournal 25:386

GiBBoN N 1958 British fournal of Urology 30:1

Ross JC 1956 Communication to the British Association of Urological Surgeons, June 1956

Ross JC 1956 British fournal of Urology 28:14

Ross JC, GibBon N, Damanski M 1957 a Lancet 2:520

Ross JC, GibBon N, Damanski M $1957 b$ Transcript of the American Association of Genito-urinary Surgery 69:193.

SHELDEN CH, BORS E 1948 fournal of Neurosurgery 5:385

WaTKINS KH 1936 British Fournal of Surgery 23:734

\section{Note}

1. A 10-years review was published in 1967 (British fournal of Surgery 54 no.7 July)

2. A twenty-years review was published in 1976 (British fournal of Urology (1976) 48:649-656)

3. A thirty-years review is in the process of preparation. 